\title{
An Online Sales System to Be Managed by People with Mental Illness
}

\author{
Alicia García-Holgado ${ }^{(\varpi)}(\mathbb{D}$, Samuel Marcos-Pablos (D), \\ and Francisco J. García-Peñalvo iD \\ GRIAL Research Group, Computer Sciences Department, Research Institute \\ for Educational Sciences, University of Salamanca, Salamanca, Spain \\ \{aliciagh, samuelmp, fgarcia\}@usal.es
}

\begin{abstract}
The percentage of the population aged 65 and over is increasing during the last decades. It is one of the problems that the European health system, and in particular the Spanish system, has to face out. This increase is linked to the rising of dependent people, whose suffer progressive deterioration of both their physical and mental capacities. In this context, technology plays a key role in improving the quality of life, not only of older people but also their caregivers. A technological ecosystem to support patients with mental illness, their caregivers, and the connection with their relatiyes was developed in previous works. This solution is prepared to evolve according to the users' and organization's needs. In this sense, the present work describes the inclusion of a new software tool, an online sales platform that promotes active ageing, seeking that it can be used and managed by older people who may have cognitive impairment problems. Although there are many e-commerce platforms on the market, they not consider users with special needs. The objective has not been to develop a software prototype from scratch, but to focus on aspects relating to accessibility and usability to improve online stores and apply these improvements to an existing solution, following the philosophy of Open Source software development. This work aims to describe the definition process itself.
\end{abstract}

Keywords: Web accessibility $\cdot$ Technological ecosystem $\cdot$ E-commerce $\cdot$ Rural areas $\cdot$ Mental health $\cdot$ Heuristic $\cdot$ Cognitive impairment

\section{Introduction}

Today it is a fact that Spain, like the rest of the European Union (EU), is ageing. According to data from the National Statistics Institute of Spain (INE), Spain registered a new ageing historical maximum in 2018, continuing with the ascending trend of the last decade. The percentage of the population aged 65 and over, which currently stands at $19.2 \%$ of the total population, is expected to rise up to $25.2 \%$ in 2033 . In this sense, and if current trends continue, the dependency ratio (quotient, as a percentage, between the population aged under 16 or over 64 and the population aged 16 to 64 ) would rise from $54.2 \%$ today to $62.4 \%$ in 2033 .

Given this reality, active ageing policies in Spain have received special attention in the last decade. Active ageing is a concept defined by the World Health Organization 
(WHO) $[1,2]$ as the process of optimizing opportunities for health, participation and safety in order to improve the quality of life as people age. In particular, in order to promote the active ageing in an environment in which the penetration of technology in different areas of life is already a reality, solutions must be proposed that allow the active participation of the older citizens in the Digital Society. In this sense, different initiatives have been promoted, like the 2011 European Agenda for Adult Learning (EAAL), which defines the focus of European cooperation on adult education policies for the period up to 2020, the Active Assisted Living Programme (AAL), or the Interuniversity Programme of Experience running since 2002-2003 in the Autonomous Region of Castile and León (Spain) [3]. The main goal is to promote ways for the senior population to acquire new job skills within the Digital Society, promoting an active lifestyle and avoiding social exclusion. However, an important aspect of older people as they age is the progressive deterioration of both their physical and mental capacities, which can make it difficult for them to use technological solutions [4].

With this in mind, a technological ecosystem [5] has been developed with two fundamental objectives. First, improving the quality of life of (in)formal caregivers through learning, ubiquitous access to information and support. Second, providing a set of services for relatives and patients with a particular focus on those who live in rural areas. Thus, the ecosystem integrates different software components with the aim of improving the welfare work based on three pillars. teaching-learning, to provide the necessary and specific training to (in)formal caregivers to provide care to the elderly; social, with the aim of sharing experiences on the process of learning and welfare work, also providing means to avoid the social exclusion of caregivers; and finally a dashboard for ecosystem management and obtaining metrics that can be used for monitoring and proposing new actions both at the welfare level and for the management of the ecosystem itself. On the other hand, and given the inherent evolutionary approach of technological ecosystems that must allow the incorporation of new components $[5,6]$, an online sales platform has been developed that promotes active ageing, seeking that it can be used and managed by older people who may have cognitive impairment problems (as well as other people with other mental illness).

In this paper, the development of the prototype of an online store that is inclusive, so that it takes into account all types of users and can also be managed by people with different abilities, particularly people with severe and prolonged mental illness, is presented. It has to be taken into account that, in the context of mental diseases, each patient has a unique clinical picture [7], so it is very difficult for two people to share the same symptoms or the same reactions to similar situations, so special care must be taken in terms of the user experience (accessibility, usability, etc.). Although there are many e-commerce platforms on the market (Etsy, Shopify, Bigcartel, Amazon, etc.), they focus on improving the usability of the system but do not consider users with special needs [8]. People with severe and prolonged mental illness must be able to manage sales or make purchases over the Internet. The aim has been to develop a system that allows a simplified sales network adapted to both workers and customers. To do this, the objective has not been to develop a software prototype from scratch, but to focus on aspects relating to accessibility and usability to improve online stores and apply these improvements to an existing solution, following the philosophy of Open Source software development. 
The rest of the paper is organized as follows. Section 2 provides an overview of the ecosystem. Section 3 describes the online sales platform. Section 4 presents the results of the heuristic evaluation of the interface for consumers. Finally, Sect. 5 summarizes the main conclusions of this work.

\section{Ecosystem Overview}

Technological ecosystems are solutions to support knowledge management in different contexts. Although in the literature are similar concepts, such as software ecosystem [9] or digital ecosystem $[10,11]$, these approaches are mainly based on a central platform to connect or develop other tools and the actors connected with the ecosystem are developers or stakeholders involved in the developing process. On the other hand, the technological ecosystem approach proposes a decentralized solution in which different software tools, human resources and information flows compose the ecosystem. Furthermore, the technological ecosystem is focused on supporting evolution in order to adapt continuously to the needs of the organization.

In this context, a technological ecosystem for (in)formal caregivers was developed with the support of the DEFINES project, "A Digital Ecosystem Framework for an Interoperable NEtwork-based Society" (Ref. TIN2016-80172-R), funding by the Spanish Ministry of Economy and Competitieness; and the TE-CUIDA project, "Technology ecosystem to support caregivers" (Ref. SA061P17), funding by the Ministry of Education of the Regional Government of Castile and León.

The ecosystem aims to develop and enhance the competences of the caregivers, both formal and informal, as well as mitigate the negative effects produced by caregiving activities such as overload, depression, or anxiety. Caregivers prevent the institutionalization of the dependent persons enabling them to stay at home and thus reducing care costs, but this has an impact on the wellbeing of caregivers, reducing their quality of life and increasing social isolation and family stress. Also, being an online solution allows access to services ubiquitously, facilitating resources such as psychoeducation, or communication with relatives to those who live in rural areas.

Any technological ecosystem is composed by three main elements [6]: software tools to provide different services within the ecosystem; human resources to ensure the evolution of the ecosystem and being directly involved in the knowledge management processes; and information flows to provide the interaction among tools, and between tools and human resources. These elements are represented in an architectural pattern and a metamodel to support the definition of technological ecosystems based on previous experiences. Both proposals, the architecture pattern and the metamodel, are the main results of the thesis dissertation [12].

The definition of the technological ecosystem for caregivers is based on those proposals. In this work, the architectural pattern is used to describe the main elements of the ecosystem (Fig. 1). The ecosystem is organized in four layers - presentation, services, static management data and infrastructure - and two input streams that introduce the human factor as another element of the technological ecosystem.

The top layer, presentation, is focused on ensuring the usability of the software components with a particular focus on user experience. Although usually this layer also 
provides a uniform interface to all the components, in this proposal, the branding associated with the ecosystem has not yet been fully implemented.

The second layer provides the software components with the main user-level services. Initially, the ecosystem consisted of three components but incorporated a fourth component, the online store. The first service is an online platform to provide a set of private and safe areas for patients, relatives and caregivers, so they can maintain the contact despite the living places or other socioeconomic situations [13]. Walls are managed by (in)formal caregivers, care managers, but also the patients and their relatives may be granted access to the social network.

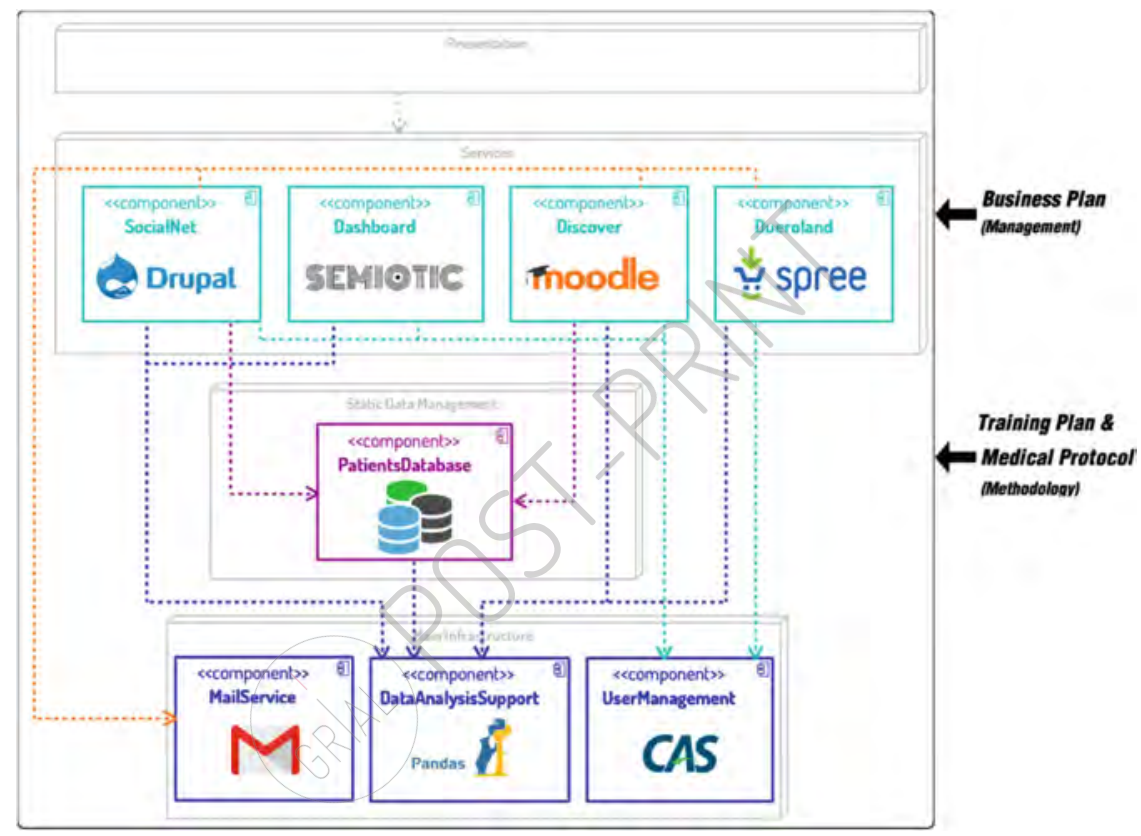

Fig. 1. The architecture of the technological ecosystem for (in)formal caregivers. Based on [14]

The second service is focused on psychoeducation $[15,16]$ for (in)formal caregivers in order to provide them with training support to cover different knowledge needs, but also information, advice, and guidance, as well as access to a community of equals and experts. The third service is a dashboard to support decision-making processes. The knowledge managed in the ecosystem is associated with different sources such as the information stored in the different tools of the ecosystem, the implicit and tacit knowledge of the users, as well as the interaction of them and the ecosystem. The dashboard combines these sources through data visualization. Finally, the fourth service is the online store described in this proposal to simplify the sales network adapted to both workers and customers with special needs. 
Regarding the static data management layer, it provides tools to centralize information needed by other components of the ecosystem. This layer has a database to store data associated with the patients: caregiving activities, provided treatments, etc.

The last layer is the infrastructure; it provides a set of services that are used by the software components from other layers. In particular, the mail server, the user management tool based on CAS (Central Authentication Service) and a tool to support data analysis as a service for the dashboard.

Finally, the human factor is represented in the architecture through two input flows: the business plan from a management point of view, and the training plan and medical protocol from a methodological perspective. Furthermore, those as sensible and medical data may be generated within the ecosystem component; the human factor should take into account the ethics and data protection necessary to warranty safe data governance [14].

\section{Online Sales System}

The online sales platform was planned to be integrated with the activities of the Special Employment Centre in Zamora (Spain) (in Spanish, Centro Especial de Empleo, CEE). The origins of the CEE are in the needs detected by professionals and associations dedicated to the rehabilitation and socio-labour reinsertion of people with disabilities due to serious and prolonged mental illness. The principal activity of the CEE consists of the creation of spaces that allow the labour integration of people with serious and prolonged mental illness, such as the design, production and marketing of products and services in which people with disabilities participate, promoting and encouraging their training and employment. Among the actiyities of the CEE in Zamora is the cultivation and marketing of organic fruit and vegetables in rural areas of Castile and León, the development of craft products with various materials, as well as cleaning and catering services.

The online sales platform aims to support the distribution of the products created in the activities associated with the CEE, so the people with mental illness will be involved not only in the production phase but also in the sales phase. Furthermore, the target audience is all types of users, but with particular emphasis on those who have some mental illness or disability.

The online sales system has been developed within the province of the technological ecosystem presented in Sect. 2. For that purpose, a similar approach as the one followed in [5], in which the authors showed the importance of modelling the business structure along with the software structure during the early stages of the ecosystem development. The main objective is not to follow a "business first" approach, but to develop the business and software structures altogether, as they are complementary. Taking into account the different business processes while developing the software structure, provides fundamental "constraints" or characteristics for the software structure, such as the data taxonomy and ontology, the data architecture, and the data security and lifecycle. 


\subsection{Business Structure Model}

As stated before, the development of the business model of the online store has started with the modelling of the business structure. To do so, Business Process Modeling Notation (BPMN) has been employed in its 2.0 version. The BPMN is a standard for business process modeling flow chart notation that models the steps of a planned business process from end to end. As such, a set of diagrams that describe the different tasks involved in the online store business processes have been obtained. They start from a high-level process conception using BPMN's Choreography diagrams that show the interaction between participants (described as senders and receivers) concentrating on the message flow. From there, more detailed Collaboration diagrams were developed that focus on the different tasks of the business to be coped with by the different participants to achieve a particular goal. Finally, lower-level business processes describing the different tasks that must be developed by the software components can be obtained. Figures 2, 3 and 4 show example instances of the modelled processes of the business, including a higher level choreography diagram for placing an order, the Collaboration diagram between the customer and the online sales system that describes the process of purchasing a product, and the Process diagram of the login task into the sales system.

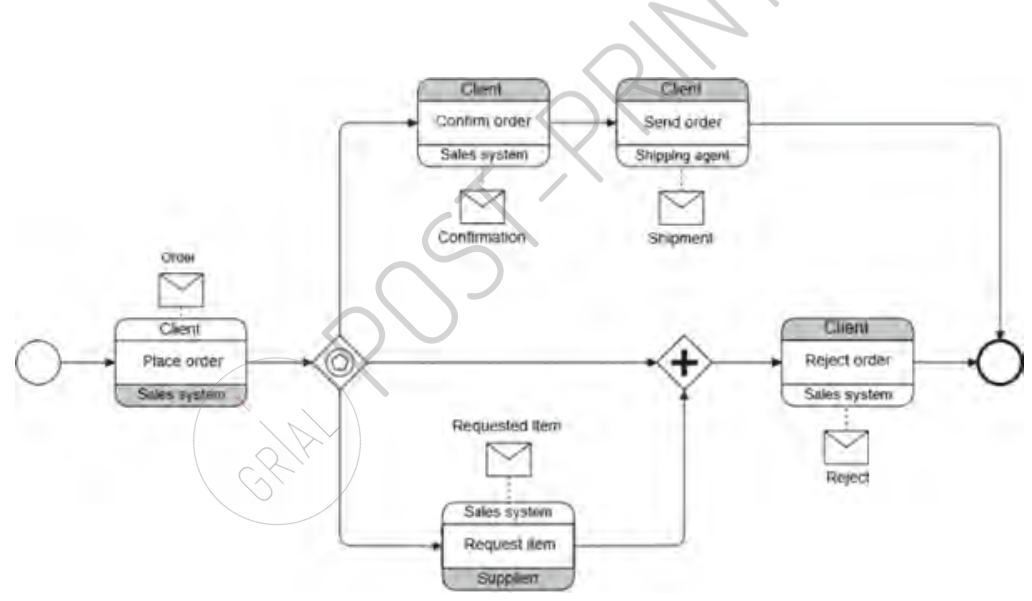

Fig. 2. BPMN 2.0 High-level choreography diagram for placing an order.

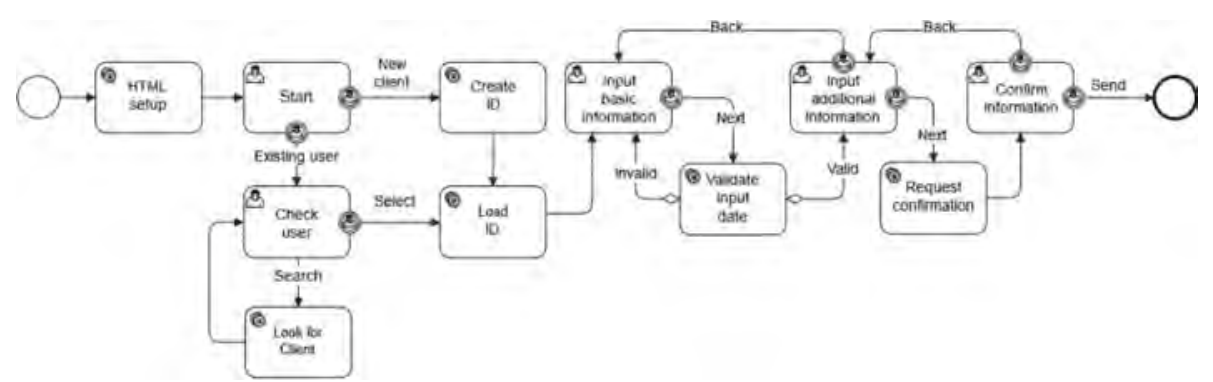

Fig. 3. BPMN 2.0 Process diagram of the login task in the sales system. 


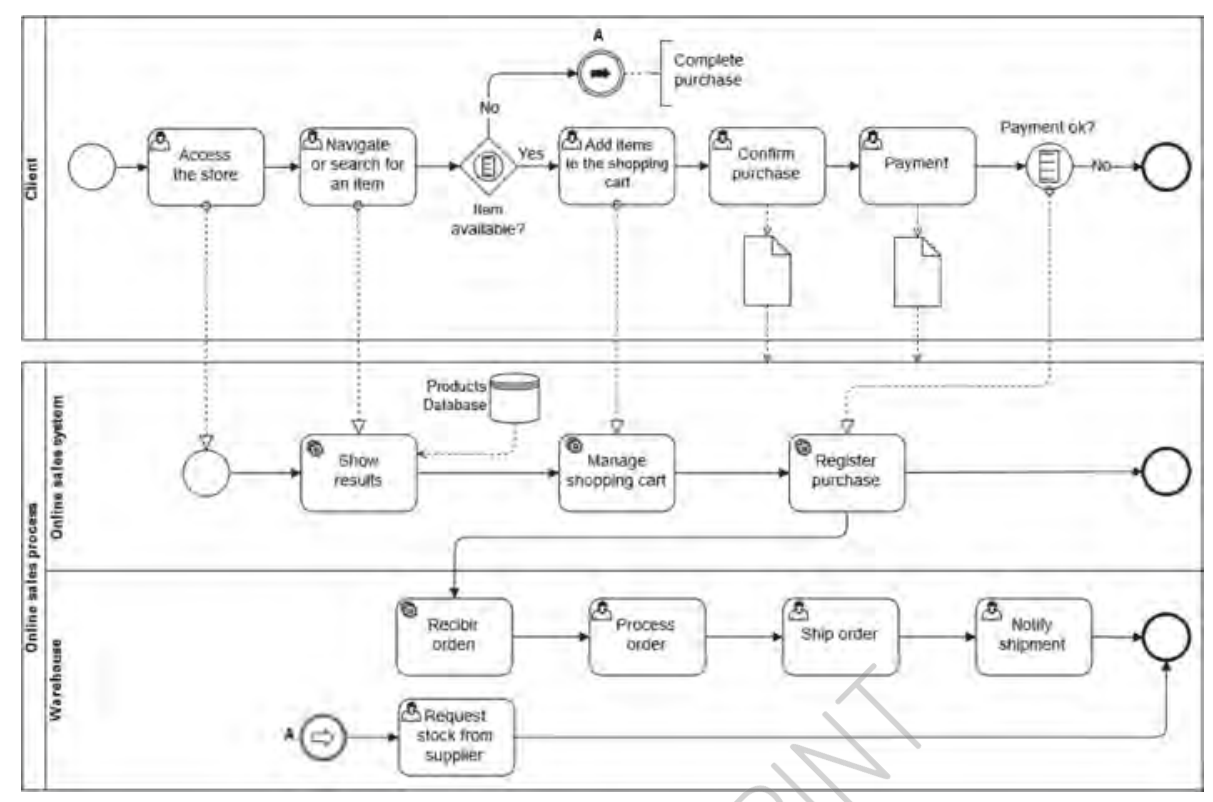

Fig. 4. BPMN 2.0 Collaboration diagram between the customer and the online sales system that describes the process of purchasing a product.

\subsection{Software Architecture}

The online store was intended to be integrated with the business management software, which was already in use by the CEE. In particular, the ERP (Enterprise Resource Planning) software used by the CEE was Navision [17]. This integration aims to facilitate the management processes associated with the store, so people with prolonged mental illness will be able to manage sales.

Based on the developed business process models, a study has been carried out of the functional and non-functional requirements of the software prototype of the online store, as well as the requirements and services necessary for its integration with the accounting and warehouse management system managed by the ERP. The online store is based on Spree Commerce, an Open source e-commerce framework developed in Ruby on Rails, which was adapted to the identified needs through the development of extensions. In particular, the integration of the online store with the ERP is done through web services under the SOAP standard, following the specifications provided by the ERP.

The functional requirements included a list and description of the different contents necessary for the development of the web services of the sales platform and their integration with the ERP: user roles (administrator, manager, client, anonymous user); data necessary for the client to register on the web (email, password, client key in Navision, etc.); product information (name, photograph, base price, description, etc.); order execution (payment method, shipping and collection method, customer number, billing data, etc.); tracking (order date, link to tracking, etc.); and others. A description 
of the different interfaces necessary for the development of the web services required to integrate these data into the ERP was also developed. On the other hand, considerations on non-functional requirements were also taken into account: integration with existing solutions, usability and internationalization.

Likewise, a document has been developed that describes, from a technical point of view, the different scenarios of the online sales system as well as its integration with the ERP. These scenarios have been subdivided into the following sections: (1) description of the scenario; (2) procedure (different steps that take place in the scenario); (3) UML sequence diagram that implements the procedure; (4) data set needed to carry out each of the ERP transactions required for each scenario.

The considered scenarios were:

- Product creation/modification: assign extra data to exist products and add them to the store or create new products (not existing products in the ERP), as well as delete products.

- Stock visualization: evaluate stock each time a customer visits a product page or tries to make a purchase.

- Sales: record sales in the ERP each time a customer buys something through the web.

- Sales Cancellation: Allow the customer to cancel a purchase (as long as the shipment has not been made) and reflect the changes in the ERP.

- From draft sale to historical sale: sales are created as drafts so that the customer can cancel them if desired. After the package has been shipped, these sales must be converted to historical.

Figure 5 shows an example of the sequence diagram for adding a new product within the product creation/modification scenario.

The next stage consisted of the visual design of the online store focused on providing a satisfying user experience, with special emphasis on users with severe and prolonged mental illness. To this end, and based on the results of the previous activities, the different screens associated with the different scenarios and components needed to meet the functional requirements have been defined, following the standards of the World Wide Web Consortium (W3C). More specifically, the store design takes into account the Web Content Accessibility Guidelines (WCAG) 2.0 standard for users with some cognitive issues [18], as well as the recommendations for the cognitive accessibility of web content available at the Web Accessibility Initiative (WAI) [19]. Although not all of the WCAG 2.0 criteria were applied, the WAI recommends meeting at least the WCAG 2.0 Level A and AA criteria, along with some Level AAA criteria that are particularly important for people with cognitive difficulties.

Finally, the connectivity of the online store with the ERP has been deployed, from which an alpha version of the sales platform has been obtained. The final prototype is available at http://dueroland.grial.eu. 


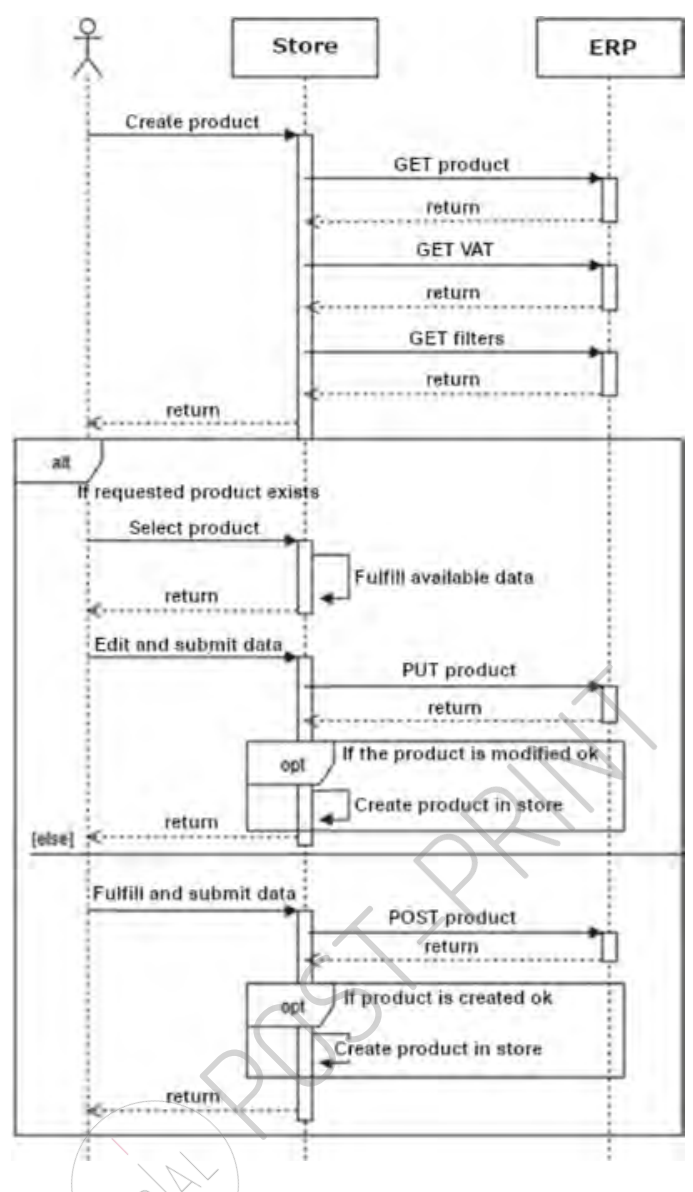

Fig. 5. UML sequence diagram for adding a product.

\section{Heuristic Evaluation}

Finally, the beta version of the platform has been developed, including in its design the aspects of usability and accessibility previously identified and proceeding to its usability study in two ways: through heuristic tests carried out by experts in usability, and through tests with the platform workers that are planned to be conducted in the future.

The first part of the usability study, the heuristic evaluation, was focused on the interface for clients. It was carried out by two experts, two men between 25 and 38 years old. None of the experts had used the online store previously. One expert was involved in the projects that support the definition and development of the system, and the other one was directly involved in the development. None of the experts has cognitive impairments or mental illness, but they have knowledge related to technology apply to mental health. In addition to these characteristics, the criteria used to select the experts was based on their professional profiles: 
- E1: A full stack developer with six years of experience developing online platforms for health and wellbeing sectors.

- E2: A researcher with more than ten years of experience in multimodal humancomputer interaction.

Each expert reviewed the online store. They identified the usability problems associated with each heuristic proposed by Nielsen [20] and assigned a value from 1 (major usability problems) to 10 (no usability problems). Table 1 summarizes the values for each heuristic rule. The average of each heuristic was calculated in order to get a final value for each heuristic, so this value reflects where are more usability issues.

Table 1. Assigned values to each heuristic by each expert

\begin{tabular}{l|r|r|l}
\hline Heuristic rule & E1 & E2 & Avg. \\
\hline HR1: Visibility of system status & 10 & 7 & 8.5 \\
\hline HR2: Match between system and the real world & 10 & 10 & 10 \\
\hline HR3: User control and freedom & 10 & 7 & 8.5 \\
\hline HR4: Consistency and standards & 10 & 5 & 7.5 \\
\hline HR5: Error prevention & 9 & 8 & 8.5 \\
\hline HR6: Recognition rather than recall & 10 & 7 & 8.5 \\
\hline HR7: Flexibility and efficiency of use & 10 & 7 & 8.5 \\
\hline HR8: Aesthetic and minimalist design & 7 & 7 & 7 \\
\hline HR9: Help users recognize, diagnose, and recover from errors & 8 & 7 & 7.5 \\
\hline HR10: Help and documentation & 4 & 6 & 5 \\
\hline & & &
\end{tabular}

\section{Discussion and Conclusions}

Active ageing is one of the main objectives of the current society. The population aged over 65 years old has increased during the last decades, and the figures will continue rising up. This situation supposes a challenge for the health systems across the world. In this context, active ageing is one of the main objectives of the World Health Organization in order to improve the quality of life as people age. This approach is combined with technology to provide solutions that allow active participation of older citizens in the Digital Society.

The current proposal aims to provide a set of guidelines to develop an inclusive online store, so that it takes into account all types of users and can also be managed by people with different abilities, particularly people with severe and prolonged mental illness, is presented. The result is a prototype of an online sales platform in which production and sales are carried out by people with mental illness.

It is important to emphasize the context in which the prototype of the online store has been deployed. Although it is an online solution, accessible from any region in the world, it is specially focused on promoting the distribution of products among the different rural areas in Zamora, in the first instance, and subsequently at the regional level, in Castile and León, and the national level, Spain. Currently, the prototype is 
operative and contains products made in the workshops and activities of the CEE, with home delivery service available for the Zamora region, including the entire metropolitan area and rural areas of the province.

The tool was correctly integrated with the ERP to manage the sales, but also it was correctly added to the technological ecosystem for (in)formal caregivers due to the architecture proposed in Fig. 1.

Regarding the heuristic evaluation, it is important to take into account the bias of this procedure. According to [21] the perception of evaluators in using this method is not consistent with the users' experience with a system. Despite this, the results obtained are useful to improve the system and prepare the next phase with real users. Experts detected problems associated with most of the heuristic rules, although there are significant differences between the experts. The most significant usability problem is associated with HR10 (Help and documentation); there is no documentation or users' support available in the online store. Besides, several problems were detected in HR8 (Aesthetic and minimalist design) by both experts, most of them related to the lack of products and images associated with the available products.

Finally, the findings of this study have a number of important implications for improving the development of e-commerce platform adapted to users with different abilities. On the other hand, future works need to be done to complete the usability study with qualitative techniques such as focus group with final users with different abilities and mental illness.

Acknowledgments. This work has been partially funded by the Spanish Ministry of Economy and Competitiveness throughout the DEFINES project (Ref. TIN2016-80172-R) and the Ministry of Education of the Junta de Castilla y León (Spain) throughout the TE-CUIDA project (Ref. SA061P17).

\section{References}

1. Kalache, A., Gatti, A.: Active ageing: a policy framework. Adv. Gerontol. Uspekhi Gerontol. Akad. Nauk. Gerontol. Obs. 11, 7-18 (2003)

2. WHO: Active Ageing: A Policy Framework. World Health Organization, Geneva (2002)

3. Cámara, C.P., Eguizábal, A.J.: Quality of university programs for older people in Spain: innovations, tendencies, and ethics in European higher education. Educ. Gerontol. 34, 328 354 (2008)

4. Stompór, M., Grodzicki, T., Stompór, T., Wordliczek, J., Dubiel, M., Kurowska, I.: Prevalence of chronic pain, particularly with neuropathic component, and its effect on overall functioning of elderly patients. Med. Sci. Monit.: Int. Med. J. Exp. Clin. Res. 25, 2695-2701 (2019)

5. García-Holgado, A., Marcos-Pablos, S., García-Peñalvo, F.J.: A model to define an eHealth technological ecosystem for caregivers. In: Rocha, Á., Adeli, H., Reis, L., Costanzo, S. (eds.) New Knowledge in Information Systems and Technologies. WorldCIST 2019. Advances in Intelligent Systems and Computing, vol. 932, pp. 422-432. Springer, Cham (2019)

6. García-Holgado, A., García-Peñalvo, F.J.: Architectural pattern to improve the definition and implementation of eLearning ecosystems. Sci. Comput. Program. 129, 20-34 (2016) 
7. Malla, A., Joober, R., Garcia, A.: "Mental illness is like any other medical illness": a critical examination of the statement and its impact on patient care and society. J. Psychiatry Neurosci.: JPN 40, 147-150 (2015)

8. Gonçalves, R., Rocha, T., Martins, J., Branco, F., Au-Yong-Oliveira, M.: Evaluation of ecommerce websites accessibility and usability: an e-commerce platform analysis with the inclusion of blind users. Univ. Access Inf. Soc. 17, 567-583 (2018)

9. Manikas, K., Hansen, K.M.: Software ecosystems - a systematic literature review. J. Syst. Softw. 86, 1294-1306 (2013)

10. Pillai, K., King, H., Ozansoy, C.: Hierarchy model to develop and simulate digital habitat ecosystem architecture. In: 2012 IEEE Student Conference on Research and Development (SCOReD). IEEE, USA (2012)

11. Ostadzadeh, S.S., Shams, F., Badie, K.: An architectural model framework to improve digital ecosystems interoperability. In: Elleithy, K., Sobh, T. (eds.) New Trends in Networking, Computing, E-learning, Systems Sciences, and Engineering. Lecture Notes in Electrical Engineering, vol. 312, pp. 513-520. Springer, Cham (2015)

12. García-Holgado, A.: Análisis de integración de soluciones basadas en software como servicio para la implantación de ecosistemas tecnológicos educativos. Programa de Doctorado en Formación en la Sociedad del Conocimiento. University of Salamanca, Salamanca, Spain (2018)

13. García-Peñalvo, F.J., Franco Martín, M., García-Holgado, A., Toribio Guzmán, J.M., Largo Antón, J., Sánchez-Gómez, M.C.: Psychiatric patients tracking through a private social network for relatives: development and pilot study J. Med. Syst. 40 (2016). Article no. 172

14. Marcos-Pablos, S., García-Holgado, A., García-Peñalvo, F.J.: Modelling the business structure of a digital health ecosystem. In: Conde-González, M.Á., Rodríguez Sedano, F.J., Fernández Llamas, C., García-Peñalvo, F.J. (eds.) Proceedings of the 7th International Conference on Technological Ecosystems for Enhancing Multiculturality, TEEM 2019, León, Spain, 16-18 October 2019, pp. 838-846. ACM, New York (2019)

15. Geldmacher, D.S., Kirson, N.Y., Birnbaum, H.G., Eapen, S., Kantor, E., Cummings, A.K., Joish, V.N.: Implications of early treatment among Medicaid patients with Alzheimer's disease. Alzheimer's Dement. 10, 214-224 (2014)

16. Ostwald, S.K., Hepburn, K.W., Caron, W., Burns, T., Mantell, R.: Reducing caregiver burden: a randomized psychoeducational intervention for caregivers of persons with dementia. Gerontologist 39, 299-309 (1999)

17. Diffenderfer, P.M., El-Assal, S.: Microsoft Dynamics NAV: Jump Start to Optimization. Vieweg+Teubner Verlag (2008)

18. W3C: Web Content Accessibility Guidelines (WCAG) 2.0 (2008)

19. Cognitive Accessibility at W3C. Web Accessibility Initiative (WAI). http://bit.ly/2QSj2sG

20. Nielsen, J.: Heuristic evaluation. In: Nielsen, J., Mack, R.L. (eds.) Usability Inspection Methods, vol. 17, pp. 25-62. Wiley, Hoboken (1994)

21. Khajouei, R., Ameri, A., Jahani, Y.: Evaluating the agreement of users with usability problems identified by heuristic evaluation. Int. J. Med. Inform. 117, 13-18 (2018) 